“(C) 2015 IEEE. Personal use of this material is permitted. Permission from IEEE must be obtained for all other uses, in any current or future media, including reprinting/republishing this material for advertising or promotional purposes, creating new collective works, for resale or redistribution to servers or lists, or reuse of any copyrighted component of this work in other works." 


\title{
Optimal Spectral Efficiency for Cooperative UWB Based On-Body Area Networks
}

\author{
Jie Ding*, Eryk Dutkiewicz*, Xiaojing Huang ${ }^{\dagger}$ \\ * Department of Engineering, Macquarie University, Australia \\ \{jie.ding1, eryk.dutkiewicz\}@ mq.edu.au \\ $\dagger$ CSIRO Computational Informatics, Australia \\ Xiaojing.Huang@csiro.au
}

\begin{abstract}
In this paper, spectral efficiency (SE) is investigated for cooperative ultra-wideband (UWB) based on-body area networks (OBANs). To optimize SE for single-relay cooperation, an equivalent generic cooperative model in UWB based OBANs is established first. With the proposed model, joint optimal relay location and power allocation for cooperation is then derived to solve the SE maximization problem. Simulation results show that direct transmission is preferable for UWB based OBANs when the transmitter and receiver are located on the same side of the human body. However, the joint optimal cooperative transmission scheme can achieve a significant improvement on SE compared with direct transmission when the transmitter and receiver are located on the different sides of the human body, which indicates that cooperation is more feasible to be applied in this case due to its robustness to the significant path loss.
\end{abstract}

\section{INTRODUCTION}

Wireless body area network (WBAN) is a promising technology that can improve healthcare quality with several lightweight sensors on/in the human body [1]. Ultra-wideband (UWB) technology has great potential for applications in WBAN, owing to its simple electronics and low power consumption, which is less likely to affect human tissue and causes interference to other medical equipment. For UWB based BANs, spectral efficiency (SE) is one of the most critical performance parameters [1] [2]. On the other hand, relay assisted cooperative communication has drawn much attention in wireless networks which can achieve a diversity gain that improves link reliability and SE [3]. However, the SE of cooperative communication is still an open issue for UWB based BANs.

For the SE of the relay assisted communications, considerable studies have been conducted in wireless sensor networks (WSNs) [4] [5]. In [4], the power allocation at the source and relay is optimized to maximize the SE in single-relay based cooperative networks. In [5], power allocation and relay selection schemes were proposed to achieve the maximum SE and minimum outage probability for multiple relays assisted cooperative networks. It reveals that the proposed schemes maintain full diversity order while greatly increasing the SE compared to conventional schemes.

Due to the unique properties of WBANs with distinct channel characteristics and very small scale compared with WSNs, the aforementioned existing schemes and results on SE in WSNs may be inadequate if they are applied to WBANs directly. Thus, it is worth investigating the spectral efficiency for cooperative transmission in WBANs. For cooperative transmission, some related studies have been conducted for WBANs [6] [7] [8]. In [6], cooperative transmission was first introduced for WBANs, where the spatial diversity gain is analyzed. In [7], the energy efficiency of cooperative transmission was studied with the constrained targeted outage probability. In [8], the energy efficiency of cooperative transmission was considered from a relay selection perspective for UWB based BANs. However, to the best of our knowledge, research on the SE optimization of relaying cooperation for WBANs has not appeared in the literature so far.

For this reason, we study the SE optimization problem in cooperative UWB based on-body area networks (UWB based OBANs) in this paper. Specifically, we seek the relay with the optimal location to achieve the maximum SE, together with the corresponding optimal power allocation. Firstly, an equivalent generic relay-based cooperative system model is proposed for UWB based OBANs. With the proposed model, the SE optimization problem is then mathematically formulated and the optimal relay location and power allocation are jointly derived to achieve the maximum SE. Simulation results show that cooperative transmission is effective in improving SE for UWB based OBANs. Though direct transmission is preferable when the transmitter and receiver are located on the same side of the human body without significant path loss, the joint optimal cooperative transmission scheme is very helpful for the robustness against the substantial path loss and able to achieve a significant improvement on SE compared with direct transmission when the transmitter and receiver are located on the different sides of the human body.

The remainder of this paper is organized as follows. In Section II, the system and channel models are briefly described. In Section III, the SE for cooperative transmission is presented and the joint optimal relay location and power allocation is derived accordingly. Simulation results are presented in Section IV and the paper is concluded in Section V.

\section{System And Channel Models}

In this paper, we consider a general UWB based OBAN system model consisting of several wearable sensor nodes 
and a body network coordinator on the body surface. The transmitter node (Source: S) monitors the physiological states of a person periodically and transmits information bits towards the coordinator (Destination: D). Then D can send them to the personal server wirelessly for further processing. As shown in Fig. 1, we consider the single relay cooperative case where an adjacent on-body node $\mathrm{R}$ (relay) can assist $\mathrm{S}$ in delivering its message to $\mathrm{D}$. In this paper, we assume that $\mathrm{R}$ and $\mathrm{S}$ are always located on the same side of the human body. With the distance $d_{s d}[\mathrm{~m}]$ between $\mathrm{S}$ and $\mathrm{D}$, we denote $d_{1}$ and $d_{2}$ as the distance from $\mathrm{S}$ to $\mathrm{R}$ and the one from $\mathrm{R}$ to $\mathrm{D}$, respectively.

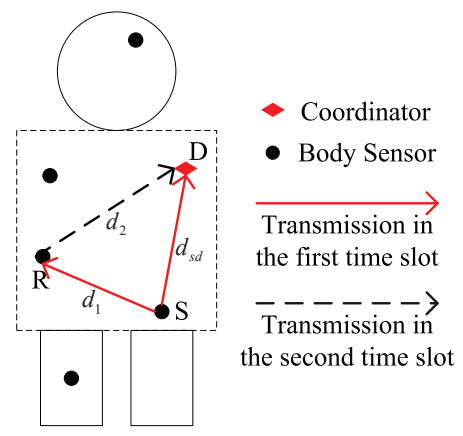

Fig. 1. A general system model for cooperative OBANs.

The cooperation protocol is a typical time division multiple access (TDMA) protocol shown in Fig. 1. It consists of two time slots with equal duration, in which $\mathrm{S}$ broadcasts its signal to $\mathrm{D}$ and $\mathrm{R}$ during the first time slot, and in the second time slot, $\mathrm{R}$ always forwards its received signal to $\mathrm{D}$ independently regardless of the results of the transmission in the first time slot, since a feedback is not available from D to R. This protocol can achieve a maximum degree of broadcasting and exhibit no receive collision. It is noted that in practice the amplify and forward (AF) relay, when compared with the decode and forward (DF) relay, requires significantly lower implementation complexity since no decoding is required at $\mathrm{R}$. Thus we only consider the AF relay in UWB based OBANs.

For cooperative transmission, two types of channel models need to be considered in UWB based OBANs, namely "along torso" path loss model and "around torso" path loss model, respectively. With a given location of D, "along torso" refers to the condition that S and D are on the same side of the human body and "around torso" refers to the condition that S and $\mathrm{D}$ are on the different sides of the human body. Table I summarizes the corresponding parameters for these two models [9] [10].

Fig. 2 compares the path loss $[\mathrm{dB}]$ for the along-torso model and around-torso model. We can see the around-torso path loss is much stronger compared to the along-torso case with the same distance. Since signals are always transmitted over the along-torso channel for the S-R link, it is indicated that cooperative transmission in the around-torso scenario may be very helpful in improving SE compared with the around-torso based direct transmission.

From the path loss models defined in log scale, we can
TABLE I

UWB BASED BAN PATH LOSS MODELS FOR ON-BODY

\begin{tabular}{|c|c|}
\hline \hline $\begin{array}{c}\text { "along torso" channel } \\
\text { model }\end{array}$ & $P L_{0}^{d B}(d)=P_{0}+10 n_{0} \log _{10}\left(\frac{d}{d_{0}}\right)$ \\
\hline$P_{0}[\mathrm{~dB}]$ & 44.6 \\
\hline$n_{0}$ & 3.1 \\
\hline$d_{0}[m]$ & 0.1 \\
\hline \hline $\begin{array}{c}\text { "around torso" channel } \\
\text { model }\end{array}$ & $P L_{1}^{d B}(d)=P_{1}+10 n_{1} \log _{10}\left(\frac{d}{d_{1}}\right)$ \\
\hline$P_{1}[\mathrm{~dB}]$ & 48.4 \\
\hline$n_{1}$ & 5.9 \\
\hline$d_{1}[m]$ & 0.1 \\
\hline
\end{tabular}

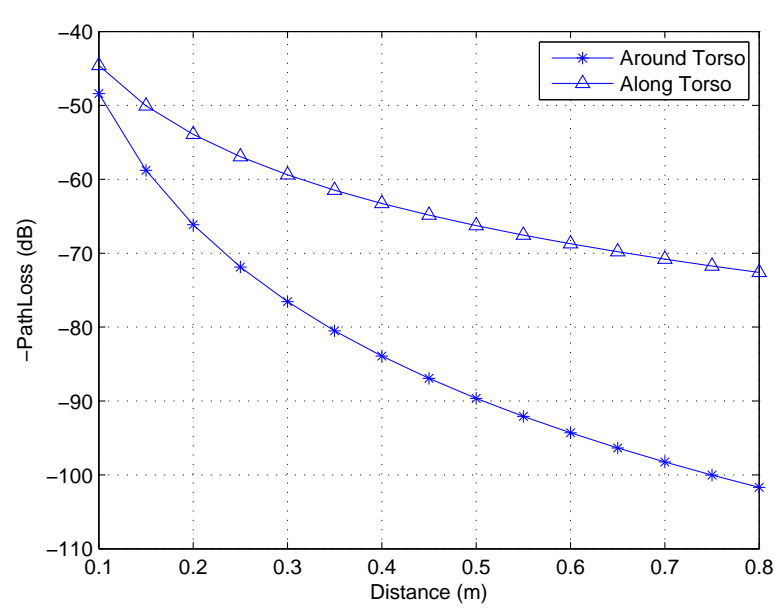

Fig. 2. Path loss $(\mathrm{dB})$ versus distance $(\mathrm{m})$ for "along torso" and "around torso".

obtain the path losses in linear scale for both scenarios as,

$$
P L_{0}(d)=10^{P L_{0}^{d B}(d) / 10}=M_{0} d^{n_{0}},
$$

and

$$
P L_{1}(d)=10^{P L_{1}^{d B}(d) / 10}=M_{1} d^{n_{1}},
$$

where $M_{0}=\left(1 / d_{0}\right)^{n_{0}} 10^{P_{0} / 10}$ and $M_{1}=\left(1 / d_{1}\right)^{n_{1}} 10^{P_{1} / 10}$ are constant.

In next Section, the SE for cooperative transmission is investigated and the joint optimal relay location and power allocation for cooperation is derived to maximize the SE.

\section{Spectral EFFiciency AnAlysis For COOPERATIVE TRANSMISSION}

\section{A. Spectral Efficiency for Direct Transmission}

$\mathrm{SE}[\mathrm{bits} / \mathrm{s} / \mathrm{Hz}]$ is one of the most critical performance parameters for designing UWB based BANs. To evaluate SE for cooperative transmission in UWB based OBANs, direct transmission is used to make a comparison. For direct transmission from $\mathrm{S}$ to $\mathrm{D}$, the corresponding $\mathrm{SE}$ is given by,

$$
r_{s d}=\log _{2}\left(1+\lambda_{s d}\right)
$$

where $\lambda_{s d}$ is the signal-to-noise ratio (SNR) of the S-D link. $\lambda_{s d}=\frac{P_{t}}{P L_{0}\left(d_{s d}\right) \sigma_{n}^{2}}$ for the along-torso scenario and $\lambda_{s d}=$ 
$\frac{P_{t}}{P L_{1}\left(d_{s d}\right) \sigma_{n}^{2}}$ for the around-torso scenario. $P_{t}$ is the direct transmission power and $\sigma_{n}^{2}$ is the additive white Gaussian noise (AWGN) power.

\section{B. Equivalent Cooperative Model for UWB based OBANs}

To evaluate the SE for cooperative transmission in UWB based OBANs, an equivalent cooperative model is developed in Fig. 3. As shown in this figure, the around-torso scenario is considered. Without loss of generality, we assume that D is located on the back side of the human body (surface1) and $\mathrm{S}$ and $\mathrm{R}$ are located on the front side of the human body (surface2). The distance between these two surfaces is $d_{r}$ $\left(d_{r} \geq 0\right)$. We denote point $\mathrm{P}$ as the projection of $\mathrm{D}$ on surface 2 and point $\mathrm{T}$ is located in the middle between $\mathrm{P}$ and $\mathrm{S}$. For surface2, we can construct a xy-plane to present the location of $\mathrm{R}$, where $\mathrm{S}$ is set to be the origin point and the $\mathrm{x}$-axis is along $\mathrm{P}$ to $\mathrm{S}$.

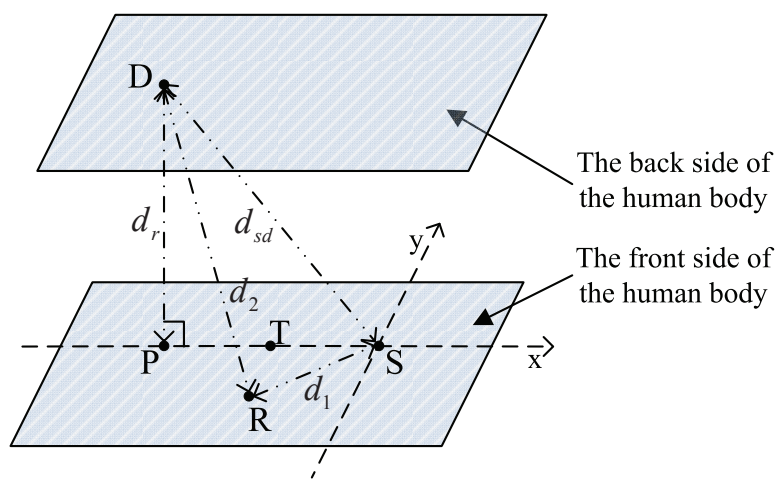

Fig. 3. An equivalent cooperative model for OBANs.

It is noted that the equivalent cooperative model will be simplified to the one for the along-torso scenario when $d_{r}=0$. In this case, D is located on the same surface with $\mathrm{S}$. Thus, we can consider the along-torso scenario as a special case in this equivalent cooperative model.

With a given $d_{s d}, d_{r}$ and the relay location $(x, y)$, we have

$$
d_{1}(x, y)=\sqrt{x^{2}+y^{2}}
$$

and

$$
d_{2}(x, y)=\sqrt{\left(x+d_{s d} \sin \theta\right)^{2}+y^{2}+d_{r}^{2}},
$$

where $\theta=\arccos \left(d_{r} / d_{s d}\right)$.

Based on the proposed model, the joint relay location and power allocation problem is mathematically formulated to maximize the related SE in the next subsection.

\section{Problem Formulation}

In cooperative transmission, with the assumed AF cooperative protocol, $\mathrm{D}$ and $\mathrm{R}$ first receive the signal from the transmitter $\mathrm{S}$ in the first time slot, and then $\mathrm{R}$, as a transmitter, forwards its received signal to $\mathrm{D}$ in the second time slot. Thus, the instantaneous received SNR at D with a maximum ratio combining (MRC) detector [8] is

$$
\lambda_{s r d}=\lambda_{1}+\lambda_{2}
$$

where $\lambda_{1}$ is the received SNR at D in the first time slot and

$$
\lambda_{2}=\frac{\lambda_{s r} \lambda_{r d}}{\lambda_{s r}+\lambda_{r d}+1} \approx \frac{\lambda_{s r} \lambda_{r d}}{\lambda_{s r}+\lambda_{r d}},
$$

is the received SNR at D in the second time slot. $\lambda_{s r}$ and $\lambda_{r d}$ are the received SNRs for link S-R and R-D, respectively.

With Equation (4), the normalized SE for cooperative transmission can be expressed as

$$
r_{s r d}=\frac{1}{2} \log _{2}\left(1+\lambda_{1}+\lambda_{2}\right)
$$

1) Around-Torso Scenario: for the around-torso scenario, the expressions of $\lambda_{1}, \lambda_{r d}$ and $\lambda_{s r}$ can be given by

$$
\begin{aligned}
& \lambda_{1}=\frac{P_{1}}{P L_{1}\left(d_{s d}\right) \sigma_{n}^{2}}, \\
& \lambda_{r d}=\frac{P_{2}}{P L_{1}\left(d_{2}\right) \sigma_{n}^{2}}, \\
& \lambda_{s r}=\frac{P_{1}}{P L_{0}\left(d_{1}\right) \sigma_{n}^{2}},
\end{aligned}
$$

and $P_{1}$ and $P_{2}$ are the transmitted power at $\mathrm{S}$ and $\mathrm{R}$, respectively. In this paper, we assume that $P_{1}+P_{2}=P_{t}, P_{1}=\alpha P_{t}$, $P_{2}=(1-\alpha) P_{t}$ and $\alpha$ is the power allocation weight [11].

Substituting Equations (2), (3) and (7) - (9) into (6), we have

$$
\begin{aligned}
r_{s r d}(x, y, \alpha) & =\frac{1}{2} \log _{2}\left(1+\frac{\alpha P_{t}}{P L_{1}\left(d_{s d}\right) \sigma_{n}^{2}}\right. \\
& \left.+\frac{1}{\frac{P L_{0}\left(d_{1}(x, y)\right) \sigma_{n}^{2}}{\alpha P_{t}}+\frac{P L_{1}\left(d_{2}(x, y)\right) \sigma_{n}^{2}}{(1-\alpha) P_{t}}}\right)
\end{aligned}
$$

where $x, y$ and $\alpha$ are variables in $r_{s r d}$. To obtain the optimal $r_{s r d}$ for cooperative transmission, we must find the optimal set $\left\{x_{o}, y_{o}, \alpha_{o}\right\}$ that makes $r_{s r d}(x, y, \alpha)$ achieve its maximum.

Thus, the joint relay location and power allocation problem for the around-torso scenario can be mathematically formulated as

$$
\begin{array}{ll}
\underset{x, y, \alpha}{\operatorname{maximize}} & r_{\text {srd }}(x, y, \alpha) \\
\text { subject to } & \mathrm{C} 1:\left(x+\frac{d_{s d} \sin \theta}{2}\right)^{2}+y^{2} \leq\left(\frac{d_{s d} \sin \theta}{2}\right)^{2}, \\
& \mathrm{C} 2: x \leq \epsilon, \\
& \mathrm{C} 3: 0 \leq \alpha \leq 1,
\end{array}
$$

where $\mathrm{C} 1$ is imposed to guarantee that $\mathrm{R}$ is only located in the circle centered at $\mathrm{T}$ with radius $\frac{d_{s d} \sin \theta}{2}$. $\mathrm{C} 2$ is imposed to guarantee that $\{x=0, y=0\}$ has to be beyond $\mathrm{C} 1$ since $\mathrm{R}$ cannot coincide with $\mathrm{S}$, where $|\epsilon|$ is a very small constant and we set $-10^{-6}<\epsilon<0$. With $\mathrm{C} 1$ and $\mathrm{C} 2$, we have $d_{1}, d_{2}<$ $d_{s d}$. 
2) Along-Torso Scenario: for the along-torso scenario, $d_{r}=0$ and the expressions of $\lambda_{1}, \lambda_{r d}$ and $\lambda_{s r}$ can be given by

$$
\begin{aligned}
& \lambda_{1}=\frac{\alpha P_{t}}{P L_{0}\left(d_{s d}\right) \sigma_{n}^{2}}, \\
& \lambda_{r d}=\frac{(1-\alpha) P_{t}}{P L_{0}\left(d_{2}\right) \sigma_{n}^{2}}, \\
& \lambda_{s r}=\frac{\alpha P_{t}}{P L_{0}\left(d_{1}\right) \sigma_{n}^{2}} .
\end{aligned}
$$

Similar to the around-torso scenario, we can express the corresponding $r_{s r d}$ as

$$
\begin{aligned}
r_{s r d}(x, y, \alpha) & =\frac{1}{2} \log _{2}\left(1+\frac{\alpha P_{t}}{P L_{0}\left(d_{s d}\right) \sigma_{n}^{2}}\right. \\
& \left.+\frac{1}{\frac{P L_{0}\left(d_{1}(x, y)\right) \sigma_{n}^{2}}{\alpha P_{t}}+\frac{P L_{0}\left(d_{2}(x, y)\right) \sigma_{n}^{2}}{(1-\alpha) P_{t}}}\right) .
\end{aligned}
$$

With Equation (15), the SE maximization problem for the along-torso scenario can be given by

$$
\begin{array}{cl}
\underset{x, y, \alpha}{\operatorname{maximize}} & r_{s r d}(x, y, \alpha) \\
\text { subject to } & \mathrm{C} 1:\left(x+\frac{d_{s d}}{2}\right)^{2}+y^{2} \leq\left(\frac{d_{s d}}{2}\right)^{2}, \\
& \mathrm{C} 2:-d_{s d}+\epsilon \leq x \leq \epsilon, \\
& \mathrm{C} 3: 0 \leq \alpha \leq 1,
\end{array}
$$

where $\mathrm{C} 2$ is imposed to guarantee that $\mathrm{R}$ cannot coincide with $\mathrm{S}$ and $\mathrm{D}$.

Since the along-torso scenario can be considered as a special case for the around-torso scenario in the proposed cooperative model, we only detail the solution of the SE maximization problem for the around-torso scenario in the next subsection.

\section{Joint Optimal Relay Location and Power Allocation for Cooperation}

Since $\log _{2}(1+x)$ is a strictly increasing function of $x$, with Equation (11), we can rewrite the optimization problem as

$$
\begin{array}{ll}
\underset{x, y, \alpha}{\operatorname{maximize}} & \lambda_{s r d}(x, y, \alpha) \\
\text { subject to } & \mathrm{C} 1, \mathrm{C} 2, \mathrm{C} 3,
\end{array}
$$

where

$$
\begin{aligned}
\lambda_{s r d}(x, y, \alpha) & =\underbrace{\frac{\alpha P_{t}}{P L_{1}\left(d_{s d}\right) \sigma_{n}^{2}}}_{\lambda_{1}(\alpha)} \\
& +\underbrace{\frac{1}{P L_{0}\left(d_{1}(x, y)\right) \sigma_{n}^{2}}+\frac{P L_{1}\left(d_{2}(x, y)\right) \sigma_{n}^{2}}{\alpha P_{t}}}_{\lambda_{2}(x, y, \alpha)} .
\end{aligned}
$$

From Equation (17), we can see that $\lambda_{1}(\alpha)$ is a linear function of $\alpha$, which indicates that $\lambda_{1}(\alpha)$ is concave. For $\lambda_{2}(x, y, \alpha)$, with Equations (2) and (3), it is easy to prove that $1 / \lambda_{2}(x, y, \alpha)$ is a convex function since its related hessian matrix is positive semidefinite with $0<\alpha<1$ (the proof of this part is omitted due to space limitations) and $1 / \lambda_{2}(x, y, \alpha)>0$. Hence, $\lambda_{2}(x, y, \alpha)$ is concave w.r.t. C1$\mathrm{C} 3$ [12]. As a result, the objective function $\lambda_{s r d}(x, y, \alpha)$ is concave. On the other hand, the inequality constraint functions $\left(x+\frac{d_{s d} \sin \theta}{2}\right)^{2}+y^{2}, x$ and $\alpha$ are convex. Thus, it is concluded that the joint relay location and power allocation problem in this paper is a nonlinear convex optimization problem, which can be solved by using the Lagrange multiplier method with Karush-Kuhn-Tucker (KKT) conditions [12] [13].

The Lagrangian of Equation (16) can be given by

$$
\begin{aligned}
& \mathcal{L}\left(x, y, \alpha, \mu_{1}, \mu_{2}, \mu_{3}, \mu_{4}\right) \\
= & \lambda_{s r d}(x, y, \alpha)-\mu_{1}\left(\left(x+\frac{d_{s d} \sin \theta}{2}\right)^{2}+y^{2}-\left(\frac{d_{s d} \sin \theta}{2}\right)^{2}\right) \\
& -\mu_{2}(x-\epsilon)-\mu_{3}(\alpha-1)+\mu_{4} \alpha,
\end{aligned}
$$

where $\mu_{1}, \mu_{2}, \mu_{3}, \mu_{4} \geq 0$ are the Lagrange multipliers connected to $\mathrm{C} 1-\mathrm{C} 3$.

Taking the stationarity condition of $\alpha$, we get

$$
\begin{aligned}
\frac{\partial \mathcal{L}}{\partial \alpha}= & \frac{P_{t}}{P L_{1}\left(d_{s d}\right) \sigma_{n}^{2}}+P_{t} \frac{(1-\alpha)^{2} A_{1}(x, y)-\alpha^{2} A_{2}(x, y)}{\left((1-\alpha) A_{1}(x, y)+\alpha A_{2}(x, y)\right)^{2}} \\
& -\mu_{3}+\mu_{4}=0,
\end{aligned}
$$

where $A_{1}(x, y)=P L_{0}\left(d_{1}(x, y)\right) \sigma_{n}^{2}$ and $A_{2}(x, y)=$ $P L_{1}\left(d_{2}(x, y)\right) \sigma_{n}^{2}<P L_{1}\left(d_{s d}\right) \sigma_{n}^{2}$ since $d_{2}(x, y)<d_{s d}$.

Considering the case that $\alpha=1$, we can set $\mu_{4}=0$ based on the KKT conditions. Substituting $\alpha=1$ into Equation (19), we get

$$
\left.\frac{\partial \mathcal{L}}{\partial \alpha}\right|_{\alpha=1}=\frac{P_{t}}{P L_{1}\left(d_{s d}\right) \sigma_{n}^{2}}-\frac{P_{t}}{P L_{1}\left(d_{2}\right) \sigma_{n}^{2}}-\mu_{3}=0 .
$$

Since $\frac{P_{t}}{P L_{1}\left(d_{s d}\right) \sigma_{n}^{2}}<\frac{P_{t}}{P L_{1}\left(d_{2}\right) \sigma_{n}^{2}}$ and $\mu_{3} \geq 0$, we have $\left.\frac{\partial \mathcal{L}}{\partial \alpha}\right|_{\alpha=1}<0$, which violates the KKT conditions. Thus we can force $\mu_{3}=0$. Similarly, it is easy to prove that $\mu_{4}=0$ since $\left.\frac{\partial \mathcal{L}}{\partial \alpha}\right|_{\alpha=0}>0$. With $\mu_{3}=\mu_{4}=0$, we can see that $0<\alpha_{o}<1$.

Taking the stationarity condition of $y$ and $x$, we get

$$
\begin{aligned}
\frac{\partial \mathcal{L}}{\partial y} & =y n_{0} M_{0} A_{3}(x, y, \alpha)+y n_{1} M_{1} A_{4}(x, y, \alpha) \\
& +y \frac{2 \mu_{1}}{\lambda_{2}^{2}(x, y, \alpha)}=0,
\end{aligned}
$$

and

$$
\begin{aligned}
\frac{\partial \mathcal{L}}{\partial x}= & x n_{0} M_{0} A_{3}(x, y, \alpha)+\left(x+d_{s d} \sin \theta\right) n_{1} M_{1} A_{4}(x, y, \alpha) \\
& +\frac{2\left(x+\frac{d_{s d} \sin \theta}{2}\right) \mu_{1}+\mu_{2}}{\lambda_{2}^{2}(x, y, \alpha)}=0
\end{aligned}
$$

where $\lambda_{2}(x, y, \alpha)>0, A_{3}(x, y, \alpha)=\frac{\sigma_{n}^{2}\left(x^{2}+y^{2}\right)^{\frac{n_{0}-2}{2}}}{P_{t} \alpha}>0$ and $A_{4}(x, y, \alpha)=\frac{\sigma_{n}^{2}\left(\left(x+d_{s d} \sin \theta\right)^{2}+y^{2}+d_{r}^{2}\right)^{\frac{n_{1}-2}{2}}}{(1-\alpha) P_{t}}>0$ with the KKT conditions for $0<\alpha<1$. Obviously, the optimal $y_{o}=0$.

Considering the case $\mu_{1} \neq 0$, we can set $\left(\tilde{x}_{o}+\frac{d_{s d} \sin \theta}{2}\right)^{2}+$ $y_{0}^{2}=\left(\frac{d_{s d} \sin \theta}{2}\right)^{2}$ and $\mu_{2}=0$ based on the KKT conditions. With $y_{o}=0, \tilde{x}_{o}=-d_{s d} \sin \theta$, and substituting $\tilde{x}_{o}$ into Equation (22), we can see that $\left.\frac{\partial \mathcal{L}}{\partial x}\right|_{\tilde{x}_{o}}<0$. Hence, we can force $\mu_{1}=0$. Similarly, it is easy to prove that $\mu_{2}=0$. 
Thus, the optimal $x_{o}$ meets

$$
\frac{x_{o} n_{0} M_{0}}{n_{1} M_{1}} A_{3}\left(x_{o}, 0, \alpha_{o}\right)+\left(x_{o}+d_{s d} \sin \theta\right) A_{4}\left(x_{o}, 0, \alpha_{o}\right)=0 \text {. }
$$

Accordingly, with Equation (19) and $y_{0}=\mu_{3}=\mu_{4}=0$, the optimal $\alpha_{o}$ meets

$$
\frac{1}{P L_{1}\left(d_{s d}\right) \sigma_{n}^{2}}+\frac{\left(1-\alpha_{o}\right)^{2} A_{1}\left(x_{o}, 0\right)-\alpha_{o}^{2} A_{2}\left(x_{o}, 0\right)}{\left(\left(1-\alpha_{o}\right) A_{1}\left(x_{o}, 0\right)+\alpha_{o} A_{2}\left(x_{o}, 0\right)\right)^{2}}=0 .
$$

With Equations (23) and (24), we can solve and get the optimal set $\left\{x_{o}, y_{o}, \alpha_{o}\right\}$ for the SE maximization problem in the around-torso scenario. For the along-torso scenario, the related optimal set can also be achieved with a similar derivation.

In next Section, we compare the SEs between direct transmission and cooperative transmission and assess the SE of the proposed cooperative transmission scheme by simulation in UWB based OBANs.

\section{Simulation}

To assess the SE of the proposed cooperative transmission scheme in UWB based OBANs, numerical results are conducted in this Section. In simulations, the noise power density is set to be $-174 \mathrm{dBm} / \mathrm{Hz}$ and the system bandwidth is $500 \mathrm{MHz}$. According to the scale of the human body, transmission distance $d_{s d}$ is very limited. In this paper, we consider the case that $0.5 \mathrm{~m} \leq d_{s d} \leq 0.8 \mathrm{~m}$.

TABLE II

OPTIMAL SET $\left\{x_{o}, y_{o}, \alpha_{o}\right\}$ FOR BOTH SCENARIOS

\begin{tabular}{|c|c|c|c|c|}
\hline \multicolumn{5}{|c|}{ Along-torso scenario $\left(d_{r}=0\right)$} \\
\hline$d_{s d}$ & $0.5 \mathrm{~m}$ & $0.6 \mathrm{~m}$ & $0.7 \mathrm{~m}$ & $0.8 \mathrm{~m}$ \\
\hline$x_{o}$ & -0.30 & -0.36 & -0.42 & -0.48 \\
\hline$y_{o}$ & 0 & 0 & 0 & 0 \\
\hline$\alpha_{o}$ & 0.70 & 0.70 & 0.70 & 0.70 \\
\hline \multicolumn{5}{|c|}{ Around-torso scenario with $d_{r}=0.2 \mathrm{~m}$} \\
\hline$d_{s d}$ & $0.5 \mathrm{~m}$ & $0.6 \mathrm{~m}$ & $0.7 \mathrm{~m}$ & $0.8 \mathrm{~m}$ \\
\hline$x_{o}$ & -0.42 & -0.52 & -0.62 & -0.72 \\
\hline$y_{o}$ & 0 & 0 & 0 & 0 \\
\hline$\alpha_{o}$ & 0.42 & 0.50 & 0.57 & 0.62 \\
\hline
\end{tabular}

Table II presents the optimal set $\left\{x_{o}, y_{o}, \alpha_{o}\right\}$ with various values of $d_{s d}$ for both scenarios. It is shown that the optimal relay is always located on the negative $\mathrm{X}$-axis for both scenarios and it is very close to the point $\mathrm{P}$ in the around-torso scenario. For the along-torso scenario, we can see that the optimal power allocation weight $\alpha_{o}$ for $\mathrm{S}$ is invariant with different $d_{s d}$. This is due to the fact that, since in the along-torso scenario, all signals are transmitted over the along-torso channels, the optimal relay location relative to $\mathrm{S}$ and $\mathrm{D}$ is unchanged when $d_{s d}$ varies. Thus $\alpha_{o}$ does not vary. Different from the alongtorso scenario, $\alpha_{o}$ varies with $d_{s d}$ in the around-torso scenario. This can be explained by the fact that signals are transmitted over the along-torso channel only for the S-R link and over the around-torso channels for the other two links. The change of $d_{s d}$ has an impact on the optimal relay location relative to $\mathrm{S}$ and $\mathrm{D}$ and $\alpha_{o}$. Moreover, with a fixed $d_{r}$ and larger $d_{s d}$, it can be observed that more power is allocated to $\mathrm{S}$ to make sure that the maximum SE can be achieved for the around-torso scenario.

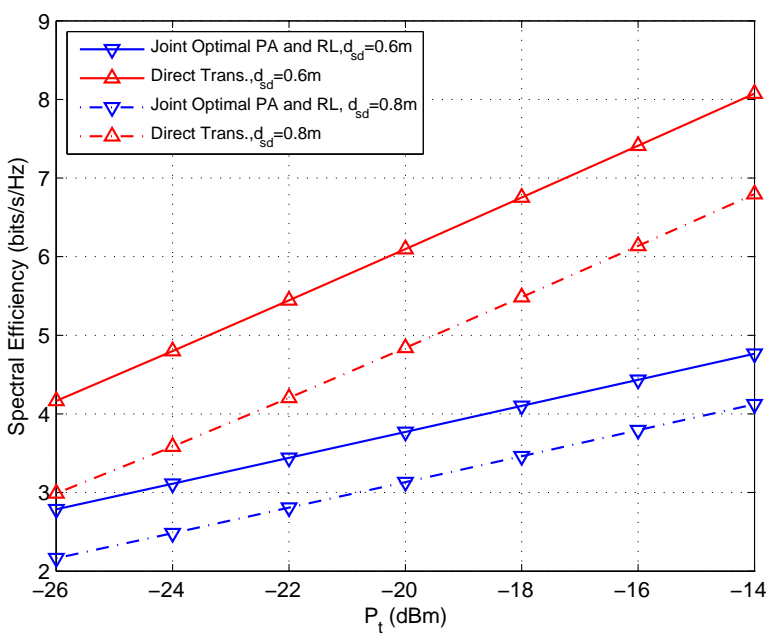

Fig. 4. SE versus $P_{t}$ for the along-torso scenario.

In Fig. 4, the spectral efficient performance is compared between direct transmission and the proposed cooperative transmission with the joint optimal power allocation (PA) and relay location (RL) for the along-torso scenario $\left(d_{r}=0\right)$. As shown in this figure, direct transmission is much more spectral efficient than the proposed cooperative scheme and the performance gap between the two schemes increases significantly as $P_{t}$ increases. This is because when a lineof-sight (LOS) between S and D is present in UWB based WBANs, the path loss exponent is small, a high SNR can be achieved by direct transmission. However, the pre-log factor $\frac{1}{2}$ in Equation (6) causes a substantial loss for cooperation in the SE in this case and this loss is especially more significant in higher SNR regime.

Fig. 5 depicts the average SE versus $P_{t}$ with different $d_{s d}$ and fixed $d_{r}=0.3 \mathrm{~m}$ for the around-torso scenario. To evaluate the performance of the joint optimal PA and RL based cooperative transmission scheme, comparisons are made with: 1) the case with equal PA and optimal RL, 2) the case with equal PA at point $\mathrm{T}$ and 3) direct transmission. It can be observed that the proposed cooperative transmission scheme can achieve the best spectral efficient performance among all the cases and all the cooperative transmission schemes outperform the direct transmission scheme. Moreover, the performance gap between the proposed cooperative transmission scheme and direct transmission is significant over a wide range of $P_{t}$ with different $d_{s d}$. We also note that, when $d_{s d}=0.8 \mathrm{~m}$, direct transmission exhibits a poor performance on SE, due to the substantial path loss. The above results indicate that cooperative transmission can achieve a remarkable improvement on SE compared with direct transmission in the around-torso scenario. Furthermore, with a higher $P_{t}$ or a larger $d_{s d}$, the improvement is more 
significant.

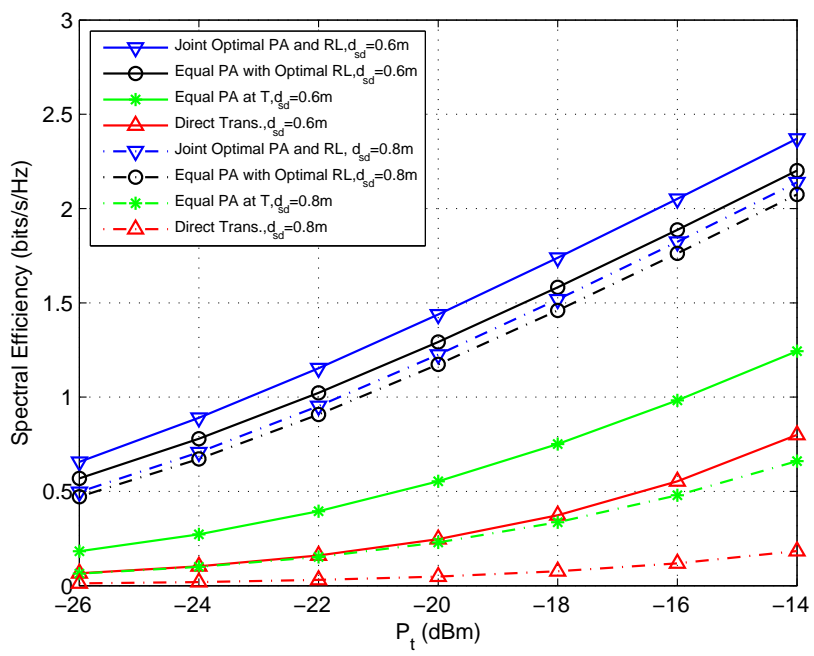

Fig. 5. SE versus $P_{t}$ in the around-torso scenario for different $d_{s d}$ and $d_{r}=0.3 \mathrm{~m}$.

Fig. 6 illustrates the average SE versus $d_{s d}$ with different $d_{r}$ and fixed $P_{t}=-15 \mathrm{dBm}$ for the around-torso scenario. Similar to Fig. 5, it is shown that the proposed cooperative transmission scheme is the most spectral efficient among all the cases. We can also notice that direct transmission is very sensitive to $d_{s d}$. That is to say, without the LOS between $\mathrm{S}$ and $\mathrm{D}$, the significant propagation loss in the around-torso scenario would affect the performance of direct transmission adversely. By contrast, the proposed cooperative transmission scheme exhibits a weak dependence upon $d_{s d}$, which indicates that the cooperation in the around-torso scenario is very helpful for the robustness against the around-torso based path loss.

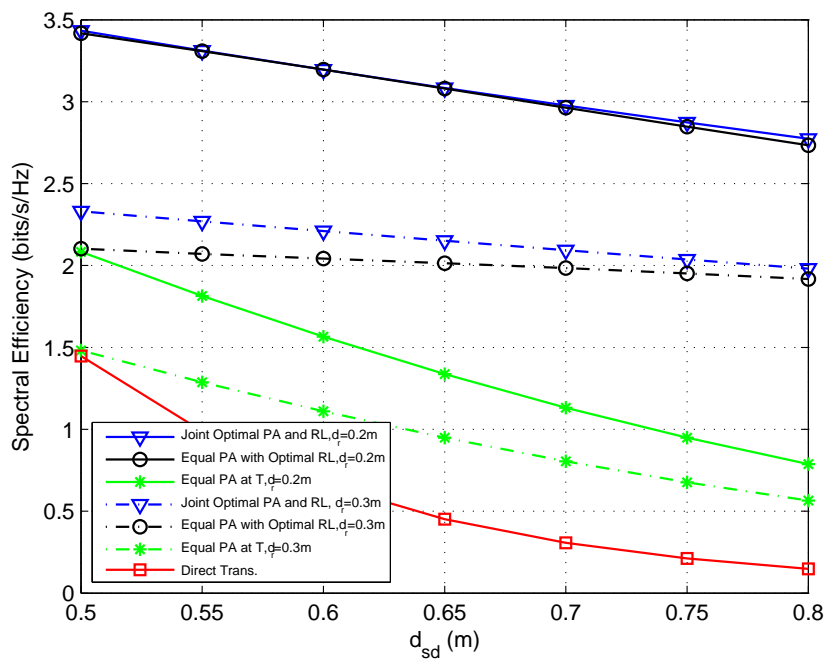

Fig. 6. SE versus $d_{s d}$ in the around-torso scenario for different $d_{r}$ and $P_{t}=-15 \mathrm{dBm}$.

In conclusion, cooperative transmission is effective in im- proving SE for UWB based OBANs, particularly in the around-torso scenario. Specifically, the proposed cooperative transmission scheme exhibits a weak dependence upon $d_{s d}$ and is robust against the substantial path loss, which is very feasible and helpful to be applied in the around-torso scenario for UWB based OBANs. With the proposed cooperative transmission scheme, a remarkable improvement on $\mathrm{SE}$ can be achieved compared with direct transmission and the improvement can be more significant with a higher $P_{t}$ or a larger $d_{s d}$.

\section{CONCLUSIONS}

In this paper, we investigated $\mathrm{SE}$ of single relay cooperative transmission for UWB based OBANs. A generic single relay based cooperative system model was proposed for UWB based OBANs. With the proposed model, the SE optimization problem was mathematically formulated and the optimal relay location and power allocation were jointly derived to maximize the corresponding SE. In the simulation, the related analysis was given and numerical results showed that cooperative transmission is effective in improving SE for UWB based OBANs. Though direct transmission is preferable for the along-torso scenario without significant path loss, the joint optimal cooperative transmission scheme is able to provide a remarkable improvement on SE compared with direct transmission for the around-torso scenario.

\section{REFERENCES}

[1] H. Cao, V. Leung, C. Chow and H. Chan, "Enabling technologies for wireless body area networks: A survey and outlook," IEEE Commun. Magazine, vol. 47, no. 12, Dec. 2009, pp. 84 - 93.

[2] M. Patel and J. Wang, "Applications, challenges, and prospective in emerging body area networking technologies," IEEE Wireless Commun., vol. 17 , no. 1,2010 , pp. $80-88$.

[3] A. Nosratinia, T. Hunter and A. Hedayat, "Cooperative communication in wireless networks," IEEE Commun. Mag., vol. 42, 2004, pp. 68 - 73.

[4] Y. Liang and V. V. Veeravalli, "Resource allocation for wireless relay channels," Asilomar Conference on Signals, Systems and Computers, Nov. 2004, pp. 1902 - 1906.

[5] Y. Zhao, R. Adve and T. J. Lim, "Improving amplify-and-forward relay networks: optimal power allocation versus selection," IEEE Trans. Wireless Commun., vol. 6, 2007, pp. 3114 - 3122.

[6] Y. Chen, J. Teo, J. C. Y. Lai, E. Gunawan, K. S. Low, C. B. Soh and P. B. Rapajic, "Cooperative communications in ultra-wideband wireless body area networks: channel modeling and system diversity analysis," IEEE J. Sel. Areas Commun., vol. 27, no. 1, Jan. 2009, pp. 5 - 16.

[7] X. G. Huang, H. G. Shan and X. M. (Sherman) Shen, "On energy efficiency of cooperative communications in wireless body area networks," IEEE WCNC, 2011, pp. 1097 - 1101

[8] J. Ding, E. Dutkiewicz, X. J. Huang, and G. F. Fang, "Energy-efficient cooperative relay selection for UWB based body area networks," IEEE ICUWB, Sep. 2013.

[9] K. Y. Yazdandoost and K. Sayrafian-Pour, "Channel model for body area network (ban)," IEEE P802.15 Working Group for Wireless Personal Area Networks (WPANs), Tech. Rep. Document IEEE802.15-08-078005-0006, February 2009.

[10] G. Dolmans and A. Fort, "Channel models WBAN-Holst centre/IMECNL," IEEE 802.15-08-0418-01-0006, July 2008.

[11] W. Su, A. K. Sadek and K. J. R. Liu, "Cooperative communication protocols in wireless networks: performance analysis and optimum power allocation," Wireless Personal Commun., vol. 44, 2008, pp. 181 - 217

[12] D. Luenberger, Linear and Nonlinear Programming: 2nd Edition. Addison-Wesley, 1984.

[13] S. Boyd and L. Vandenberghe, Convex Optimization. Cambridge University Press, 2004. 